\title{
Pretreatment with lidocaine reduces both incidence and severity of etomidate-induced myoclonus: a meta-analysis of randomized controlled trials
}

This article was published in the following Dove Press journal:

Drug Design, Development and Therapy

\author{
Bingchen Lang' \\ Lingli Zhang ${ }^{1-3}$ \\ Chunsong Yang' \\ Yunzhu Lin' \\ Wensheng Zhang ${ }^{4}$ \\ Fengshan $\mathrm{Li}^{\prime}$
}

'Department of Pharmacy, West China Second University Hospital, Sichuan University, Chengdu, People's Republic of China; ${ }^{2}$ Key Laboratory of Birth Defects and Related Diseases of Women and Children, Sichuan University, Ministry of Education, Chengdu, People's Republic of China; ${ }^{3}$ Evidence-Based Pharmacy Center, West China Second University Hospital, Sichuan University, Chengdu, People's Republic of China; ${ }^{4}$ Laboratory of Anesthesia and Critical Care Medicine, Translational Neuroscience Center, West China Hospital, Sichuan University, Chengdu, People's Republic of China

Correspondence: Lingli Zhang Key Laboratory of Birth Defects and Related Diseases of Women and Children, Ministry of Education, West China Second University Hospital, Sichuan University, No 20, Section 3, Renmin Nanlu, Chengdu 6I004I, Sichuan, People's Republic of China

Tel +8628 85503220

$\mathrm{Fax}+862885503220$

Email zhlingli@sina.com

\begin{abstract}
Objective: One conundrum that frequently occurs during clinical anesthesia is etomidateinduced myoclonus, which results in multiple risks. The aim of the study was to evaluate systematically the effect of pretreatment with lidocaine on preventing etomidate-induced myoclonus.

Materials and methods: The literature search was performed from the inception to April 2018 in PubMed, Embase, Cochrane Library, and China National Knowledge Infrastructure. All randomized controlled trials that used lidocaine to prevent etomidate-induced myoclonus were enrolled. The primary outcome included the incidence and severity of etomidate-induced myoclonus. The data were combined to calculate the risk ratio and relevant $95 \%$ CI. A metaanalysis was performed following the guidelines of the Cochrane Reviewer's Handbook and the Preferred Reporting Items for Systematic Reviews and Meta Analyses statement.

Results: A total of eight studies were enrolled, and the existing evidence indicated that 1) pretreatment with lidocaine can reduce the incidence of etomidate-induced myoclonus (the incidence of myoclonus: $37.6 \%$ in lidocaine vs $73.6 \%$ in saline, risk ratio $=0.46$, with $95 \% \mathrm{CI}[0.34$, 0.63 ],$P<0.0001)$; 2) the pretreatment with lidocaine can reduce the incidence of mild, moderate, and severe myoclonus; 3 ) a dose of pretreatment with lidocaine cannot significantly decrease the duration of myoclonus compared to placebo; 4) the administration of lidocaine produced no effect on the stable hemodynamic parameters and no more additional adverse effects.

Conclusion: Pretreatment with lidocaine could be served as one effective approach to decrease both the incidence and the severity of etomidate-induced myoclonus, with limited influence on the hemodynamic stability of patients. However, to confirm precise safety and efficacy of such intervention, more high-quality evidence is necessary.
\end{abstract}

Keywords: lidocaine, etomidate, myoclonus, meta-analysis

\section{Introduction}

Owing to its distinctive advantages including limited effect on circulation and less breathing suppression, etomidate, as one of the commonly used intravenous sedatives, provides hemodynamic stability and the smooth induction of anesthesia. ${ }^{1}$ However, problems of using etomidate such as suppression of adrenal function, injection pain, and myoclonus arouse wide concern globally, ${ }^{2-4}$ especially about the involuntary myoclonus that emerges during the induction of anesthesia that $80 \%$ of patients have experienced, ${ }^{4}$ which may lead to muscle damage, myalgia, and accidental dislodgement of the vascular access and monitoring devices. ${ }^{5}$ Although the issues of adrenocortical 
suppression and injection pain are resolved separately by synthesis of rapidly metabolized soft analogs $\mathrm{s}^{6,7}$ and formulation optimization, both the mechanism of myoclonus and the reasonable solution to it are still unclear.

Various pharmacological approaches such as opioids, benzodiazepines, $\alpha 2$ agonists, $N$-methyl-D-aspartate antagonists, muscle relaxants, and lidocaine have been reported to attenuate the etomidate-induced myoclonus. ${ }^{8-13}$ An ideal pretreatment should be short acting and have limited effects on stable hemodynamics and respiration. Recently, increasing studies have demonstrated the efficacy of lidocaine in preventing myoclonus, but a relevant systematic review has not been established. The present meta-analysis therefore was systematically conducted with the aim to evaluate the effect of pretreatment with lidocaine.

\section{Materials and methods}

This meta-analysis was performed in accordance with the recommendations in the Preferred Reporting Items for Systematic Reviews and Meta Analyses statement ${ }^{14}$ and the guidelines described in the Cochrane Handbook.

\section{Inclusion and exclusion criteria}

\section{Participants}

Adult patients with American Society of Anesthesiologists (ASA) physical status I or II who underwent general anesthesia induced with etomidate were included.

\section{Interventions and comparisons}

The intervention was pretreatment with lidocaine for preventing etomidate-induced myoclonus; the control group was given saline placebo.

\section{Outcome measurements}

The primary outcome was the number of patients reporting any myoclonus (incidence of myoclonus emergence); if the measurement scores among different studies were similar, the severity of myoclonus (the incidence of etomidate-induced myoclonus at different degrees) would also be evaluated.

The secondary outcome was the duration of myoclonus, and the mean arterial blood pressure (MAP), heart rate (HR), and the adverse effects observed in patients were also reviewed if the enrolled clinical studies mentioned these data.

\section{Studies}

Randomized controlled trials (RCTs) with no language limitations were included. Trials were excluded if the data were from case reports, reviews, or animal studies, or if the pretreatment did not involve lidocaine.

\section{Search strategy}

The literature search for published RCTs was conducted by two reviewers (BL and CY), and the databases included PubMed, Embase, Cochrane Library, and China National Knowledge Infrastructure.

The search terms: "Etomidate," "Hypnomidate," "myoclonus," "myoclonic movement," "fasciculation," "twitching," "lidocaine," "lignocaine" were combined in the search for relevant studies. The search was restricted to human studies, and the language of publications was not restricted. The last literature search was performed on April 17, 2018.

\section{Literature screening and data extraction}

Two reviewers independently screened literature and extracted data and then cross-checked with each other. After screening of the titles and abstracts, full articles were obtained when information could not be ascertained. The information derived from the literature was collected and entered in a table that included the following data: the general characteristics of studies, the types and general characteristics of patients, the sample size, and interventions and comparisons (Table 1). Disagreements were resolved by consensus through discussion among all authors.

\section{Quality assessment}

BL and CY independently evaluated the methodological quality of included studies by using the Cochrane Collaboration tool for assessing risk of bias in randomized trials. ${ }^{15}$ There are seven items by which to assess random sequence generation, including allocation concealment, blinding of participants and personnel, blinding of outcome assessment, incomplete outcome data, selective reporting and other bias, using high, low, or unclear risk of bias. ${ }^{16}$

\section{Statistics analysis}

All statistical analyses were performed using the Review Manager 5.0 software (The Cochrane Collaboration, London, UK). Dichotomous data were analyzed by using the risk ratio (RR) with 95\% CI, the Mantel-Haenszel method (fixed or random models). The $I$-squared $\left(I^{2}\right)$ test was performed to assess the impact of study heterogeneity on the results of the meta-analysis. According to the Cochrane review guidelines, if severe heterogeneity was present at $I^{2}>50 \%$, the randomeffects model was chosen; otherwise, the fixed-effects model was used. Moreover, sensitivity analysis was conducted by 


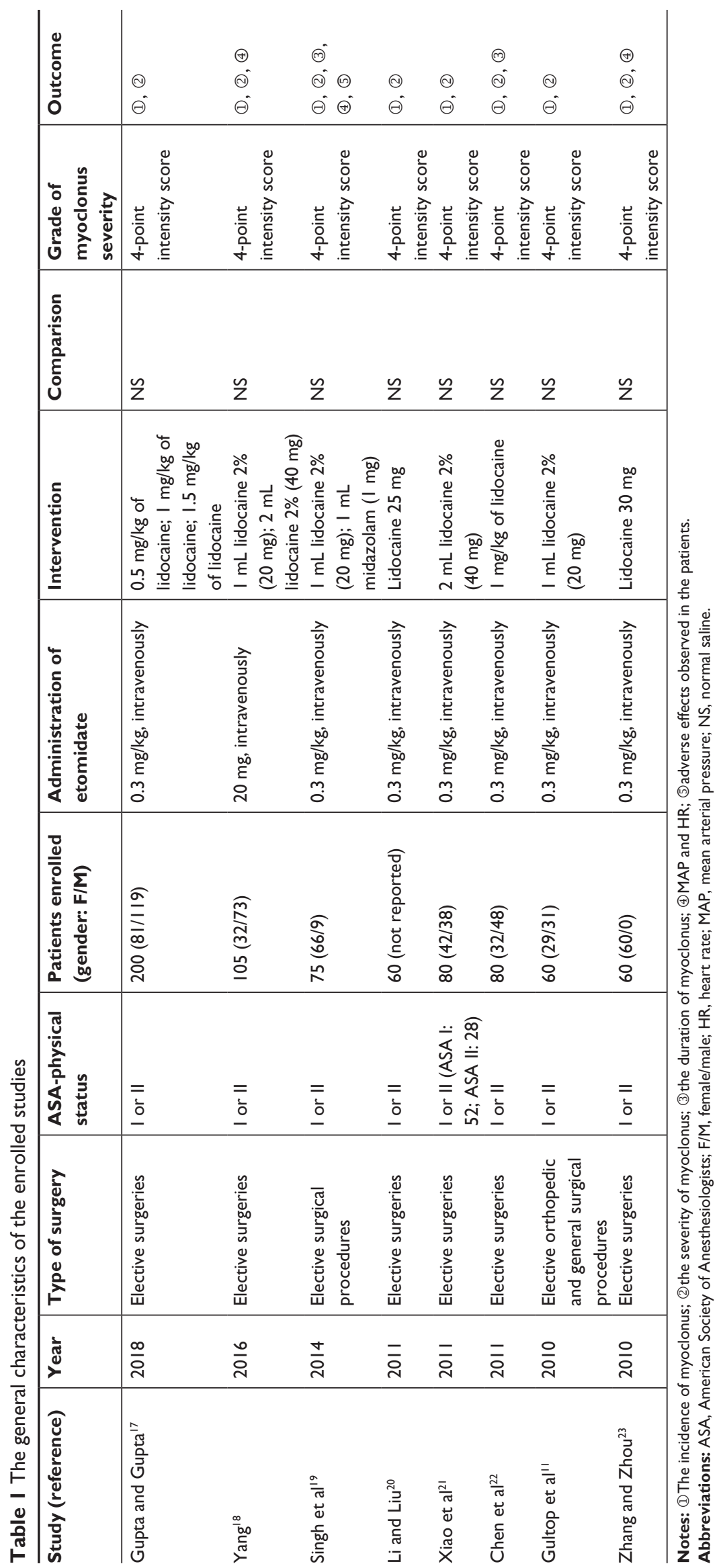


deleting each study individually to evaluate the quality and consistency of the results.

\section{Results}

\section{Literature search results}

After the search of the databases, 30 articles were identified by initial screening. Fourteen articles were excluded after duplicate removing, and one article was excluded after title and abstract review. Following the full-text review, eight RCTs were enrolled in the present systematic review. ${ }^{11,17-23}$ The identification procedure of these eligible articles is described in Figure 1. The studies from different regions were published from 2010 to 2018 .

\section{Basic characteristics of enrolled studies}

The incidence of etomidate-induced myoclonus and the severity of myoclonus were reported in all the studies. The duration of myoclonus was reported in two of them; ${ }^{19,22}$ MAP and HR were reported in two of them; ${ }^{18,23}$ and adverse effects,

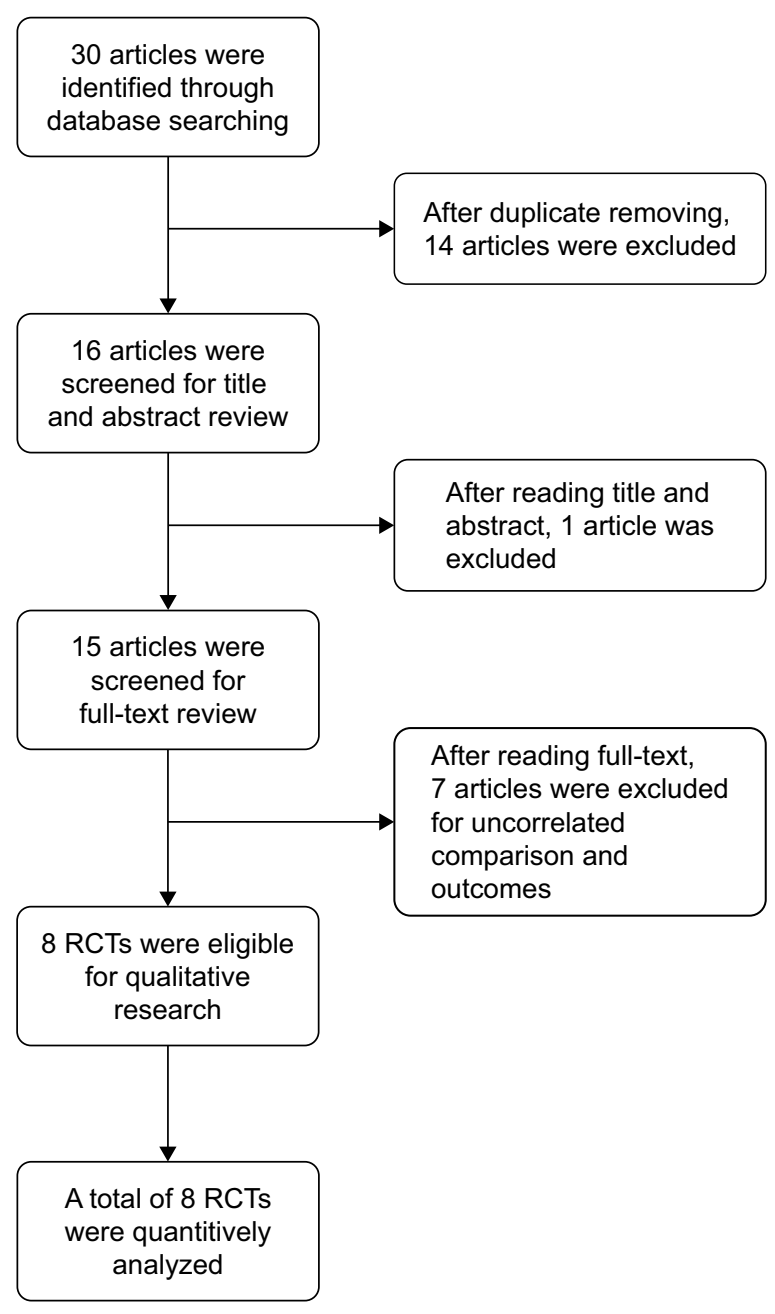

Figure I Flow chart of literature screening and the selection process. Abbreviation: RCT, randomized controlled trial. including postoperative nausea and vomiting, myalgia, and headache, were described in only one study. ${ }^{19}$ The severity of etomidate-induced myoclonus was graded on a 4-point intensity score ${ }^{4,24}$ in all of these studies. The general characteristics of the enrolled studies are shown in Table 1.

\section{Quality assessment}

According to the Cochrane Collaboration tool for assessing risk of bias, the seven items, including random sequence generation, allocation concealment, blinding of participants and personnel, blinding of outcome assessment, incomplete outcome data, selective reporting and other bias of these, including RCTs, were evaluated. A total of $25 \%(2 / 8)$ of the studies used an adequate method of random sequence generation, ${ }^{17,19}$ and only one study ${ }^{11}$ mentioned allocation concealment with the description of using opaque, sealed envelopes. Four studies did not mention the blinding procedure of participants and personnel, ${ }^{18,21-23}$ and five studies did not mention the blinding procedure of outcome assessment. ${ }^{17,18,21-23}$ The risk of bias assessment tool is shown in Figure 2.

\section{Incidence of etomidate-induced myoclonus}

All RCTs involving 695 patients were included in the study; 415 of them were given lidocaine to alleviate the myoclonus produced by using etomidate. The random-effects model was chosen due to the existence of statistical heterogeneity. Compared with the placebo (saline), the intervention of lidocaine definitely reduced the incidence of etomidateinduced myoclonus, which was described as the occurrence of myoclonus (incidence of myoclonus: $37.6 \%$ with lidocaine vs $73.6 \%$ with saline, $\mathrm{RR}=0.46$, with $95 \% \mathrm{CI}[0.34,0.63$ ], $\left.P<0.0001, I^{2}=72 \%\right)$. The $I^{2}$ of $72 \%$ indicated substantial heterogeneity, but attempts to decrease it to below $50 \%$ by excluding a single study were not successful. The results showed that lidocaine in combination with etomidate has a significantly lower incidence of myoclonus than with etomidate alone. The result is shown in Figure 3.

\section{Severity of etomidate-induced myoclonus}

The severity of myoclonus was judged visually by an observer. To standardize the observational data, each myoclonus was graded as mild, moderate, and severe. A mild myoclonus was a short movement of a body segment (a finger or a shoulder). A moderate myoclonus was slight movement of two different muscles or muscle groups of the body (face and leg). Severe myoclonus was intense clonic movement in two or more muscle groups (fast abduction of a limb). ${ }^{24}$ 


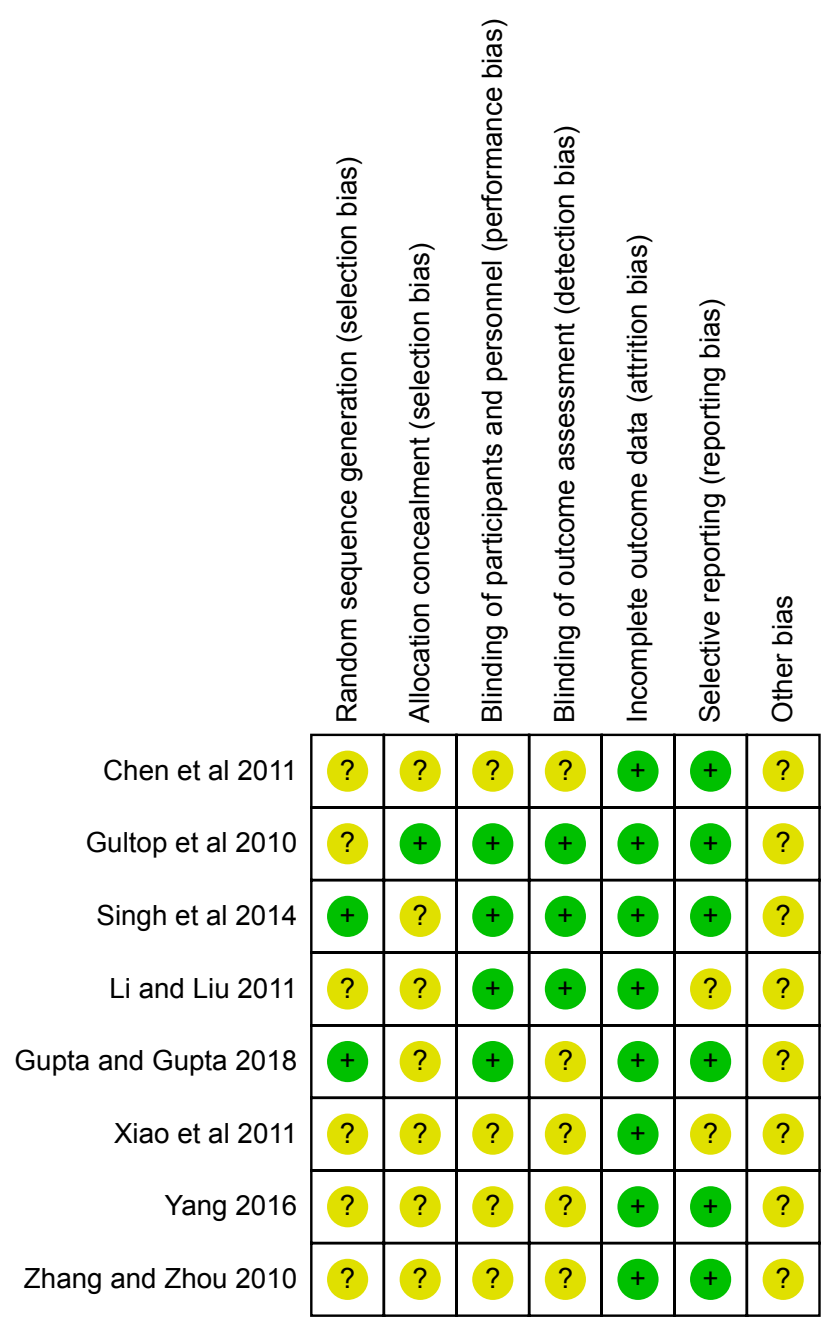

Figure 2 Risk of bias assessment of included studies.

Notes: Green + dot, low risk of bias; yellow ? dot, unclear risk of bias; red - dot, high risk of bias.

\section{Mild myoclonus}

All RCTs, involving 695 patients, were included in the study, and 415 of them were given lidocaine to alleviate the myoclonus produced by using etomidate. It was revealed that the statistical heterogeneity did not exist among the study results $\left(I^{2}=1 \%\right)$; thus, a fixed-effects model was employed to perform the analysis. The results indicated that there was a significant difference between the lidocaine group and the placebo control group in the incidence of etomidate-induced mild myoclonus (incidence of etomidate-induced mild myoclonus: $13.0 \%$ with lidocaine vs $21.1 \%$ with saline, $R R=0.62$, with $95 \%$ CI $\left.[0.44,0.88], P=0.008, I^{2}=1 \%\right)$. The results showed that lidocaine in combination with etomidate yielded a significantly lower incidence of mild myoclonus than with etomidate alone. The details are shown in Figure 4.

\section{Moderate myoclonus}

All RCTs, involving 695 patients, were included in the study, and 415 of them were pretreated with lidocaine. The fixedeffects model was chosen because statistical heterogeneity did not exist. The results indicated that the intervention of lidocaine definitely reduced the incidence of etomidateinduced moderate myoclonus (the incidence of etomidateinduced moderate myoclonus: $13.2 \%$ with lidocaine vs $28.2 \%$ with saline, $\mathrm{RR}=0.48$ with $95 \% \mathrm{CI}[0.29,0.80], P=0.004$, $I^{2}=44 \%$ ). The results (Figure 5) demonstrated that pretreatment with lidocaine produces a significantly lower incidence of moderate myoclonus.

\section{Severe myoclonus}

All the studies reported the degrees of etomidate-induced severe myoclonus. No statistical heterogeneity was found among the study results ( $\left.I^{2}=0 \%\right)$; thus, a fixed-effects model was chosen to perform the analysis, implying that there was a significant difference between the lidocaine group and the control group in the incidence of etomidate-induced severe myoclonus (incidence of etomidate-induced mild myoclonus: $11.3 \%$ with lidocaine vs $24.3 \%$ with saline, $R R=0.39$ with $95 \%$ CI $[0.28,0.55], P<0.00001$ ) (Figure 6).

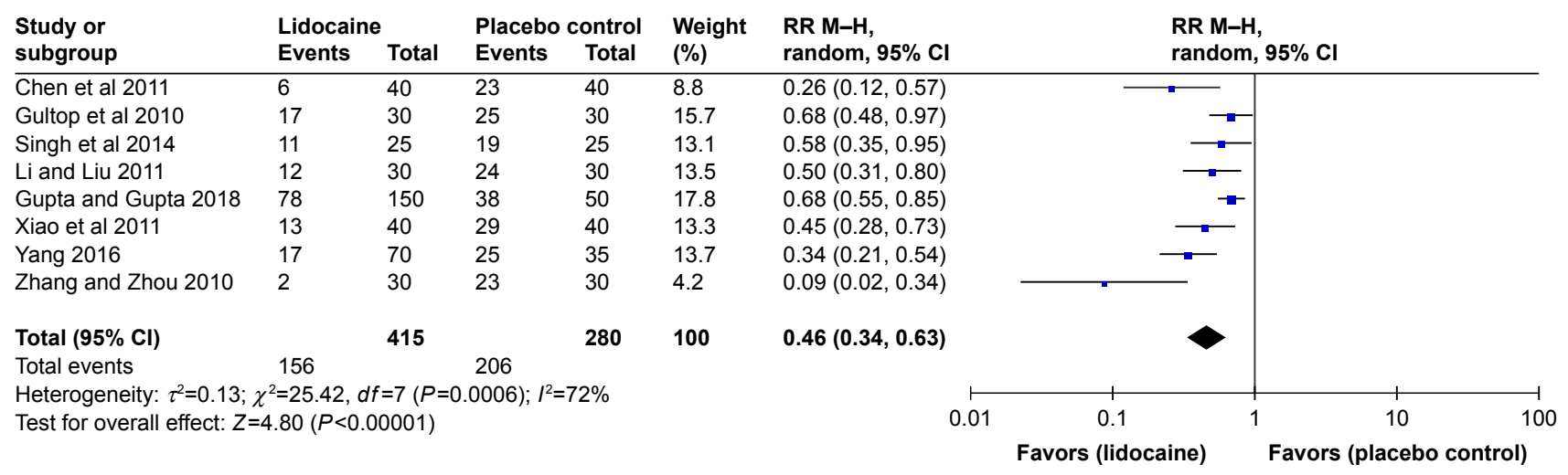

Figure 3 Forest plot of weighed RR.

Note: The lidocaine group and saline control group in the prevention of etomidate-induced myoclonus with a $95 \% \mathrm{Cl}$.

Abbreviation: RR, risk ratio. 


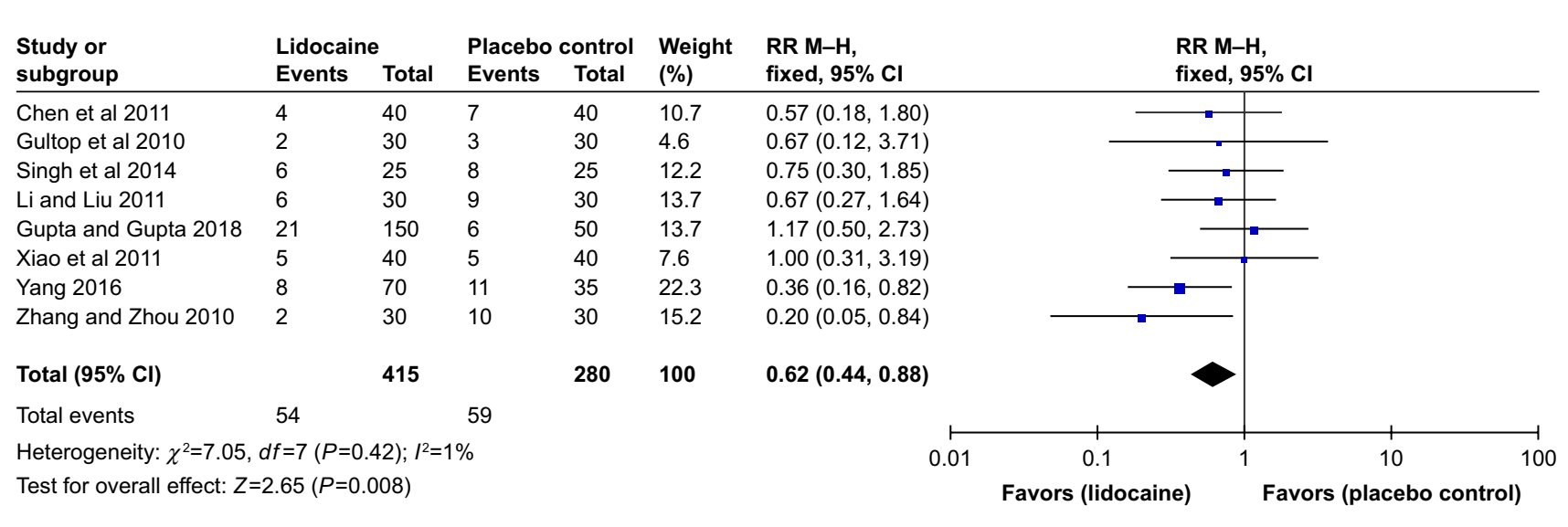

Figure 4 Forest plot of weighed RR.

Note: Results of the lidocaine group and saline control group in terms of reduction of the severity of etomidate-induced myoclonus with a $95 \% \mathrm{Cl}$ : mild myoclonus.

Abbreviation: RR, risk ratio.

\section{The duration of myoclonus}

Only two studies, ${ }^{19,22}$ involving 130 patients, reported the duration of etomidate-induced myoclonus. Based on the data from those studies, pretreatment with a dose of pretreated lidocaine did not significantly decrease the duration of myoclonus compared to placebo (weighted mean difference -11.06 minutes with $95 \%$ CI $[-35.89,13.78]$, $P=0.38$ ). However, the $I^{2}$ of $99 \%$ indicated substantial heterogeneity, but the source could not be clearly attributed to a single study, and the limited number of involved studies showed the unreliability of the result (Figure 7).

\section{Hemodynamic parameters and adverse effects}

Three studies ${ }^{18,19,23}$ described the changes in HR and MAP. In fact, the limited difference between the lidocaine and control groups indicated that the pretreatment with lidocaine produced no effect on the stable hemodynamic parameters featured by the use of etomidate. Only one study ${ }^{19}$ reported the incidence of postoperative nausea and vomiting, myalgia, and headache. Ten patients who received etomidate alone and eight patients who were pretreated with lidocaine suffered postoperative nausea and vomiting. Myalgia occurred in four patients (two received etomidate alone, and two received lidocaine pretreatment), and headache occurred in five patients, (four received etomidate alone, and only one received lidocaine pretreatment).

\section{Publication bias}

Our review included too few studies to construct a reliable funnel plot. Although there was little variation in study size, studies reported both positive and negative effects, which is not suggestive of publication bias.

\section{Discussion}

Myoclonus was defined as an involuntary, short contraction of some muscle fibers of a whole muscle, or of different muscles of one group, leading to a short, observable movement of

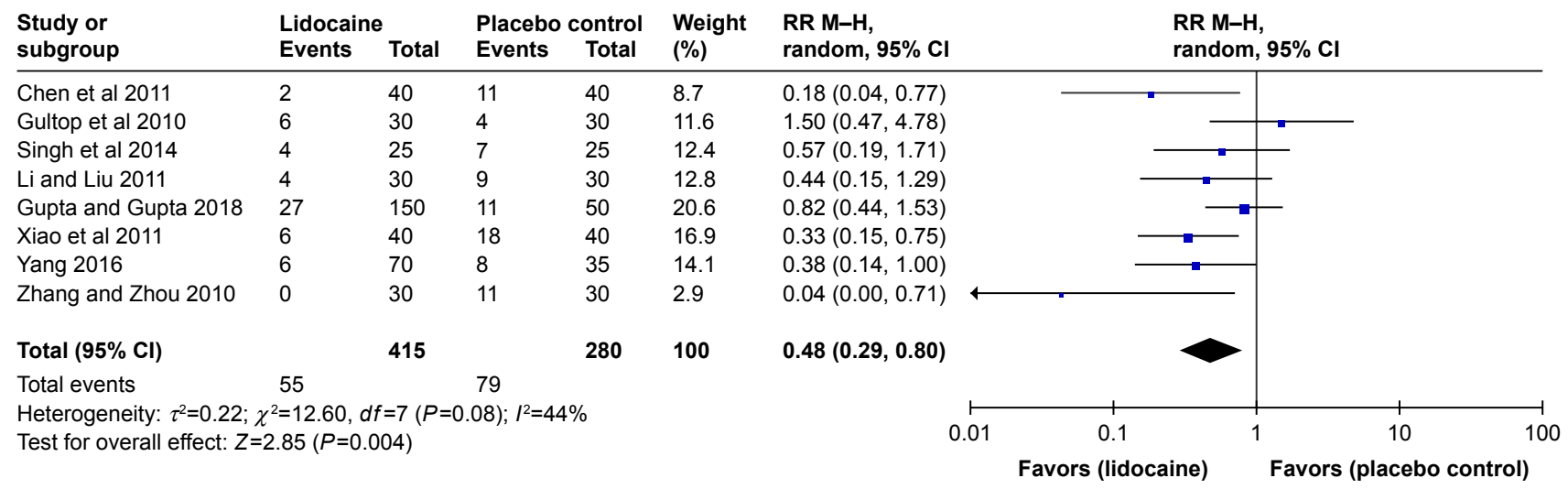

Figure 5 Forest plot of weighed RR.

Note: Results of the lidocaine group and saline control group in terms of the reduction of the severity of etomidate-induced myoclonus with a $95 \% \mathrm{Cl}$ : moderate myoclonus. Abbreviation: RR, risk ratio. 


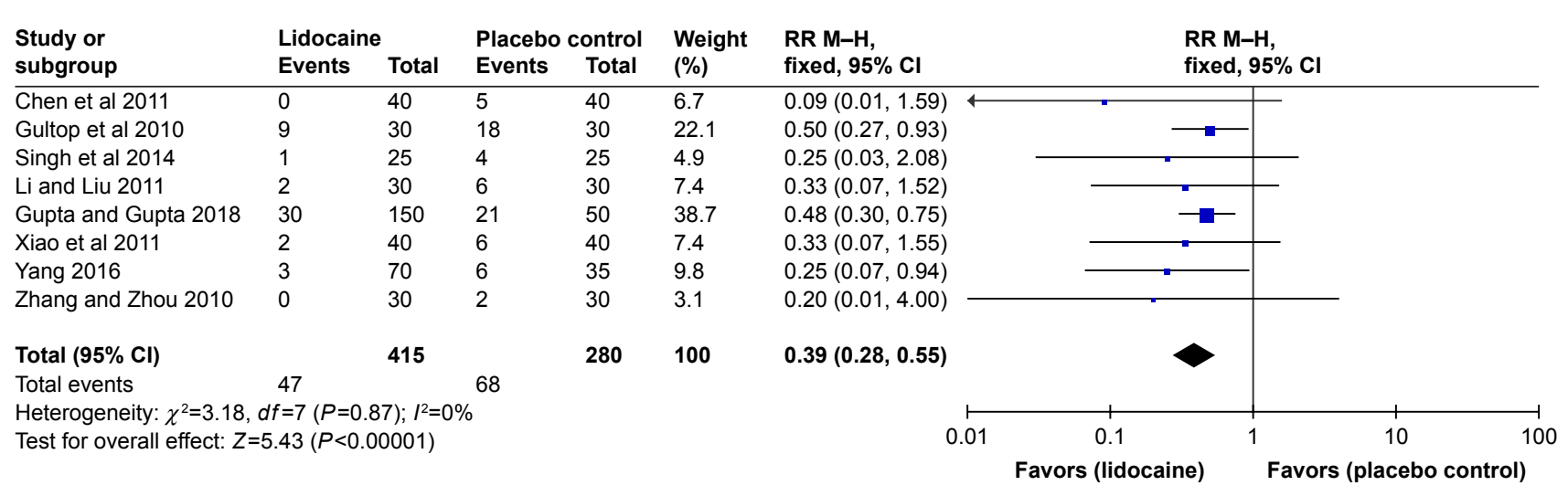

Figure 6 Forest plot of weighed RR.

Note: Results of the lidocaine group and saline control group in terms of reduction of the severity of etomidate-induced myoclonus with a $95 \%$ Cl: severe myoclonus. Abbreviation: RR, risk ratio.

the corresponding body part, usually not longer than $100 \mathrm{~ms}^{24}$ When anesthesia was induced with etomidate, $50 \%-80 \%$ of patients who were not premedicated experienced myoclonus. As previously stated, myoclonus may lead to risks to muscles and may interfere with the clinical evaluation of the depth of anesthesia.

The present study was a meta-analysis to evaluate the efficacy and safety of pretreatment with lidocaine to prevent etomidate-induced myoclonus. Based on the existing evidence from eight RCTs, the analysis indicated that lidocaine could reduce both the occurrence and the severity of etomidate-induced myoclonus, shown by the lower incidence of etomidate-induced mild, moderate, and severe myoclonus in the lidocaine pretreatment group. In addition, according to the description in relevant studies, no significant difference was found between the lidocaine group and the control group in the changes of HR and MAP, and no significant difference was found in the number of adverse effects occurrences, including postoperative nausea and vomiting, myalgia, and headache. Therefore, the preadministration of lidocaine produced limited effects on the stable hemodynamic parameters and no additional adverse effects.

The potential mechanism of myoclonus resulting from etomidate use is still unclear. Many reports have linked such involuntary movements either to a seizure-like activity or disinhibition phenomenon with earlier suppression of the cortical before subcortical activity. ${ }^{25,26}$ Disruption of the cortical GABA-mediated inhibition makes skeletal muscles susceptible to the spontaneous nerve transmissions, thereby leading to myoclonic movements, ${ }^{27}$ and the capability of lidocaine to reduce the central nervous system excitability has been hypothesized as the mechanism behind the action of myoclonus prevention. ${ }^{17}$

To our knowledge, there are no systematic reviews to summarize the efficacy and safety of pretreatment with lidocaine to prevent the etomidate-induced myoclonus. The present study was the first to review the relevant RCTs.

The limitations of the present study derived from sample sizes, quality, and design of the included trials. First, the evidence about pretreatment with lidocaine to prevent etomidate-induced myoclonus was relatively deficient when compared to the enormous amount of published literatures about interventions such as dexmedetomidine, midazolam, and opioids. In view of the previous report, the use of midazolam was more effective than lidocaine in decreasing the incidence and severity of myoclonus due to etomidate, whereas pretreatment with lidocaine produced less incidence of postoperative nausea and vomiting and myalgia. ${ }^{19}$

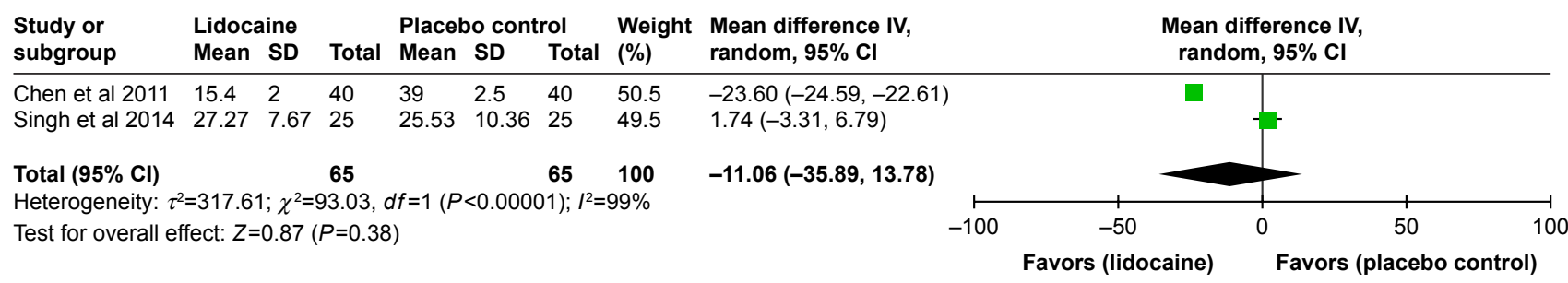

Figure 7 Forest plot of weighed RR.

Note: The effect of lidocaine in reducing the duration of myoclonus.

Abbreviation: $R R$, risk ratio. 
Because too few studies reported the comparisons among the different interventions, we were not able to evaluate the differences of the outcomes meaningfully. Second, the sample sizes of those enrolled studies varied. For instance, the recent research by Gupta and Gupta ${ }^{17}$ enrolled a significantly great number of patients than earlier studies. Thus, the results from the analysis may be distorted on some level. In addition, little published literature was aimed at the direct comparisons between different dosages of lidocaine. The search results of the databases now show only two studies that describe the effects of using different dosages of lidocaine. ${ }^{17,18}$ Considering the entirely different dosage calculation method, data combination was not performed.

\section{Conclusion}

Pretreatment of lidocaine could be served as one effective approach to decrease both the incidence and the severity of etomidate-induced myoclonus, with limited influence on the hemodynamic stability of patients. However, more evidence with high quality data and large sample size is necessary to determine the preferred pharmacological option and the proper prophylactic dosage of lidocaine. It remains an open question for further study.

\section{Disclosure}

The authors report no conflicts of interest in this work.

\section{References}

1. Forman SA. Clinical and molecular pharmacology of etomidate. Anesthesiology. 2011;114(3):695-707.

2. Chan CM, Mitchell AL, Shorr AF. Etomidate is associated with mortality and adrenal insufficiency in sepsis: a meta-analysis*. Crit Care Med. 2012;40(11):2945-2953.

3. Nyman Y, Von Hofsten K, Palm C, Eksborg S, Lönnqvist PA. EtomidateLipuro is associated with considerably less injection pain in children compared with propofol with added lidocaine. Br J Anaesth. 2006; 97(4):536-539.

4. Doenicke AW, Roizen MF, Kugler J, Kroll H, Foss J, Ostwald P. Reducing myoclonus after etomidate. Anesthesiology. 1999;90(1):113-119.

5. Du X, Zhou C, Pan L, Li C. Effect of dexmedetomidine in preventing etomidate-induced myoclonus: a meta-analysis. Drug Des Devel Ther. 2017;11:365-370.

6. Cotten JF, Husain SS, Forman SA, et al. Methoxycarbonyl-etomidate: a novel rapidly metabolized and ultra-short-acting etomidate analogue that does not produce prolonged adrenocortical suppression. Anesthesiology. 2009;111(2):240-249.

7. Wang B, Yang J, Chen J, et al. An Etomidate Analogue With Less Adrenocortical Suppression, Stable Hemodynamics, and Improved Behavioral Recovery in Rats. Anesth Analg. 2017;125(2):442-450.
8. Kelsaka E, Karakaya D, Sarihasan B, Baris S. Remifentanil pretreatment reduces myoclonus after etomidate. J Clin Anesth. 2006;18(2):83-86.

9. Zhao X, Bao R, Zhu J. Pretreatment with butorphanol reduces myoclonus after etomidate. $J$ Anesthesiol Clin Sci. 2013;2:2.

10. Un B, Ceyhan D, Yelken B. Prevention of etomidate-related myoclonus in anesthetic induction by pretreatment with magnesium. J Res Med Sci. 2011;16(11):1490-1494.

11. Gultop F, Akkaya T, Bedirli N, Gumus H. Lidocaine pretreatment reduces the frequency and severity of myoclonus induced by etomidate. $J$ Anesth. 2010;24(2):300-302.

12. Van Keulen SG, Burton JH. Myoclonus associated with etomidate for ED procedural sedation and analgesia. Am J Emerg Med. 2003; 21(7):556-558.

13. Zhu Y, Yang Y, Zhou C, Bao Z. Yuting Yang, Chengmao Zhou, Zeqing Bao. Using dezocine to prevent etomidate-induced myoclonus: a meta-analysis of randomized trials. Drug Des Devel Ther. 2017;11: 2163-2170.

14. Moher D, Liberati A, Tetzlaff J, Altman DG; PRISMA Group. Preferred reporting items for systematic reviews and meta-analyses. The PRISMA statement. BMJ. 2009;339:b2535.

15. Higgins JP, Altman DG, Gøtzsche PC, et al; Cochrane Statistical Methods Group. The Cochrane Collaboration's tool for assessing risk of bias in randomised trials. BMJ. 2011;343:d5928.

16. Higgins JP, Altman DG, Sterne JA, editors. Chapter 8: Assessing risk of bias in included studies. Cochrane Handbook for Systematic Reviews of Interventions. London: The Cochrane Collaboration; 2008:187-241.

17. Gupta P, Gupta M. Comparison of different doses of intravenous lignocaine on etomidate-induced myoclonus: a prospective randomised and placebo-controlled study. Indian J Anaesth. 2018;62(2):121-126.

18. Yang XL. Effect of different doses of lidocaine on etomidate-induced myoclonus during general anesthesia induction. Journal of Hainan Medical University. 2016;22(7):92-96.

19. Singh K, Ruchi G, Singh A, Kaur B. Efficacy of lignocaine versus midazolam in controlling etomidate-induced myoclonus: a randomized placebo-controlled study. Ain-Shams J Anaesthesiol. 2014;7(3): 460-464.

20. Li YC, Liu G. [The effect of lidocaine pretreatment on etomidateinduced myoclonus]. Guide of China Medicine. 2011;9(34):127-128. Chinese.

21. Xiao D, Cheng ZG, Kong GY, Pan BB, Liu JS. [Prevention of pretreatment with Lidocaine on myoclonus during anesthesia induction with Etomidate]. China Journal of Modern Medicine. 2011; 21(23):2915-2917. Chinese.

22. Chen Y, Chen HR, Xie LB, Xu XB, Li J, Liu YQ. [The effect of lidocaine on etomidate-induced myoclonus by etomidate during the induction of general anesthesia]. Beijing Med. 2011;33(8):642. Chinese.

23. Zhang F, Zhou Y. [Effect of lidocaine on etomidate-induced myoclonus]. Chin Hosp Pharm J. 2013;2010(30(23). Chinese.

24. Holdcroft A, Morgan M, Whitwam JG, Lumley J. Effect of dose and premedication on induction complications with etomidate. BrJAnaesth. 1976;48(3):199-205.

25. Voss LJ, Sleigh JW, Barnard JP, Kirsch HE. The howling cortex: seizures and general anesthetic drugs. Anesth Analg. 2008;107(5):1689-1703.

26. Herrera-Peco I, Wix-Ramos R, Domínguez-Gadea L, et al. Changes in cerebral perfusion induced by etomidate in patients with temporal lobe epilepsy. Rev Neurol. 2009;49(11):561-565. Spanish.

27. Sedighinejad A, Naderi Nabi B, Haghighi M, et al. Comparison of the effects of low-dose midazolam, magnesium sulfate, remifentanil and low-dose etomidate on prevention of etomidate-induced myoclonus in orthopedic surgeries. Anesth Pain Med. 2016;6(2):e35333. 


\section{Publish your work in this journal}

Drug Design, Development and Therapy is an international, peerreviewed open-access journal that spans the spectrum of drug design and development through to clinical applications. Clinical outcomes, patient safety, and programs for the development and effective, safe, and sustained use of medicines are the features of the journal, which has also been accepted for indexing on PubMed Central. The manuscript management system is completely online and includes a very quick and fair peer-review system, which is all easy to use. Visit http://www.dovepress.com/testimonials.php to read real quotes from published authors.

Submit your manuscript here: http://www.dovepress.com/drug-design-development-and-therapy-journal 